\title{
Prevalence and frequency of microembolic signals in 105 patients with extracranial carotid artery occlusive disease
}

\author{
Dirk W Droste, Ralf Dittrich, Vendel Kemény, Gernot Schulte-Altedorneburg, \\ E Bernd Ringelstein
}

\begin{abstract}
Besides the established factors "presence of symptoms" and "degree of stenosis", plaque echolucency is considered to be associated with increased risk of stroke in patients with carotid artery disease. An evaluation was carried out as to whether the prevalence and number of microembolic signals (MES) detected by transcranial Doppler ultrasound were higher in patients with echolucent carotid plaques.

One hundred and five patients with carotid artery stenosis from $20 \%-99 \%$ or occlusion underwent clinical investigations, duplex ultrasound of the carotid arteries, and a 1 hour recording from the middle cerebral artery downstream to the carotid artery pathology using the four gate technique. The presence of MES was more frequent and the number greater in symptomatic patients ( 21 out of 64 patients (33\%); mean number of MES in all 64 patients 3.1) than in asymptomatic patients (four out of 41 patients $(10 \%)$; mean number of MES in all 41 patients $0.3)(p=0.007$, and $p=0.006$, respectively). Echogenicity of the lesions did not affect either number or presence of MES. Positivity for MES and the number of MES increased with increasing degree of stenosis (both $p=0.002$ ). Four out of 12 patients with carotid artery occlusion showed MES. No MES could be detected in carotid artery stenosis below $80 \%$. There was a decline in positivity of MES and of the number of MES with the time after the ischaemic event. After 80 days or more after the index event, only one patient showed MES.
\end{abstract}

In conclusion, increasing degree of stenosis and presence of symptoms similarly affect macroembolic and microembolic risk. Thus MES may be a surrogate parameter for risk of stroke. The presence of MES in a few asymptomatic patients suggests that clinically silent circulating microemboli may give additional information on the pending embolic potential of carotid artery stenoses. Echolucency of the plaque was not related to an increased number of MES.

(F Neurol Neurosurg Psychiatry 1999;67:525-528)

Keywords: ultrasonics; carotid artery disease; embolism

In extracranial carotid artery stenosis, besides the presence of symptoms and the degree of stenosis, low plaque echogenecity has been claimed to be also associated with future stroke risk. ${ }^{1-6}$

Clinically silent circulating cerebral microemboli can be detected as high pitched signals within the transcranial Doppler (TCD) frequency spectrum. ${ }^{7}$ The presence of such microembolic signals (MES) proves ongoing embolisation into the cerebral arteries, gives information on the frequency of embolisation, and helps to localise the embolic source.

In this study we evaluated the clinical and the ultrasonic features of patients with different degrees of extracranial carotid artery occlusive disease in relation to the presence and frequency of circulating microemboli in the dependent middle cerebral artery (MCA) using the four gate technique. ${ }^{9}$ In particular, we wanted to show that the above established risk factors for macroembolisation (presence of symptoms and degree of stenosis) and the debated risk factor "echolucency" are also risk factors for microembolisation.

Patients and methods

One hundred and five patients, aged from 29 to 86 years, were investigated (mean age 62 (SD 11 years) There were 27 women and 78 men. They were inpatients and outpatients of our department and underwent routine clinical investigations. All of them had extracranial carotid artery stenosis $\geqslant 20 \%$ or internal carotid artery (ICA) occlusion on ultrasound. Sixty four of them had presented at least one transient or permanent ipsilateral ocular or cerebral ischaemic event in the carotid artery territory under investigation. The time between the recording and the last event varied from 0 to 3474 days. Five patients were in atrial fibrillation during the recording. Thirty two patients were taking intravenous heparin, one 


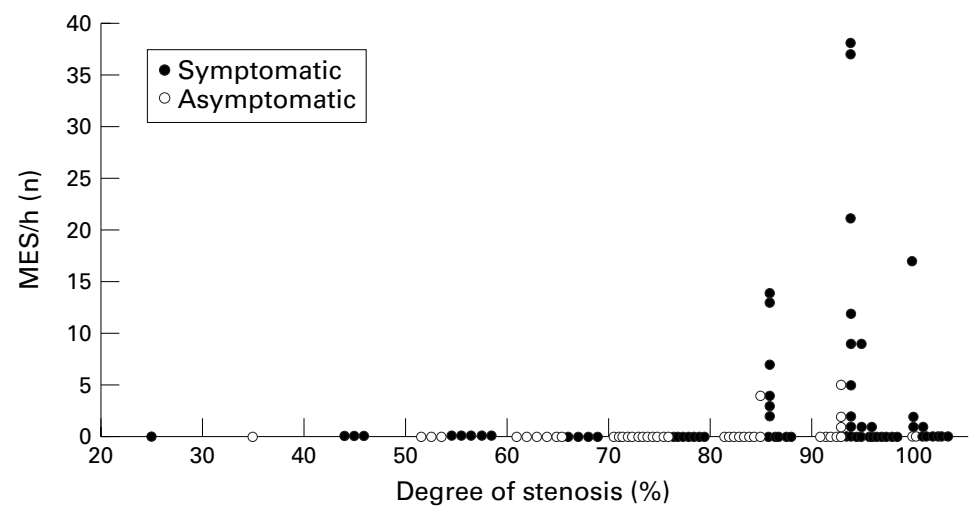

Relation between the number of MES and the degree of stenosis. There is an increasing number of MES and of MES positive patients with increasing degree of stenosis and a peak at $90 \%-99 \%$ stenosis. ies taking into account that only a small percentage of the natural fluctuations of the Doppler spectrum occurred in this range. Details of the technique are described elsewhere. ${ }^{9}$ In addition, the audio Doppler signal of all four channels was continuously recorded onto an eight channel digital audio tape deck recorder (TA-88, TEAC Corporation) with normal speed.

An experienced observer's analysis of MES consisted of (1) on line analysis during the recording, (2) visual and acoustic off line verification of the events preselected by the algorithm, and (3) off line analysis of the tapes. During the analysis of the tapes, the investigator was blinded to the information regarding the degree of stenosis, the presence of symptoms, and the echogenicity of the plaque. Conflicting results were re-evaluated and discussed.

patient subcutaneous heparin, eight oral antiaggregants, and 72 patients were taking aspirin or ticlopidine (some were on two drugs or switched, the previous drug still being active).

\section{ULTRASOUND INVESTIGATIONS}

In all patients the neck arteries were investigated by color duplex ultrasound $(7.5 \mathrm{MHz}$ linear probe, Sonos 2500, Hewlett Packard) and the periorbital arteries by continuous wave Doppler (8 MHz probe, Multidop X, DWL). The extracranial duplex investigation included longitudinal and transverse sections of the plaque and was recorded onto video tape. Furthermore the peak systolic and end diastolic flow velocities in the common carotid artery, in the jet of the stenosis, and in the most distal part of the ICA accessible by B-mode were recorded (angle adjusted). Patients were also examined by TCD (2 MHz probe, Multidop $\mathrm{X}, \mathrm{DWL}$ ).

The carotid plaque was classified as follows: (1) uniformly echolucent, (2) predominantly echolucent, (3) predominantly echogenic, (4) uniformly echogenic. ${ }^{110}$ The stenosis was classified in categories of $10 \%$ taking into account the peak systolic velocity in the jet of the stenosis, broadening of the poststenotic spectrum, peak systolic velocity in the poststenotic ICA, direction of ophthalmic flow, presence of collateral flow via communicating arteries, asymmetry in pulsatility, and absolute velocity in the common carotid artery and in the MCA. A peak systolic velocity of at least $120 \mathrm{~cm} / \mathrm{s}$ was the threshold for a stenosis $\geqslant 50 \%$ excluding subtotal stenosis with a variable signal. In the case of indirect haemodynamic criteria, the stenosis was classified to have $\geqslant 80 \%$. Occlusion was diagnosed in the complete absence of detectable flow in and above the stenosis and the presence of corresponding indirect haemodynamic criteria. Plaques with stenosis $<50 \%$ were classified according to their lumen reduction on B-mode. ${ }^{11-13}$

For the TCD embolus detection, the MCA main stem downstream to the affected carotid artery was insonated through the temporal window for 1 hour. For all studies, the same transcranial pulsed Doppler ultrasound device (TC4040, Nicolet-EME, Kleinostheim) with a $2 \mathrm{MHz}$ four gate transducer was used. A detection threshold of $\geqslant 5 \mathrm{~dB}$ was used for all stud-

\section{ANALYSIS AND STATISTICS}

The number of MES as well as the number of embolus positive patients in symptomatic patients and asymptomatic carotid artery disease were compared using the nonparametric Mann-Whitney $U$ test. The number and presence of MES were investigated in the four echogenicity categories by a KruskalWallis one way analysis of variance (ANOVA). The impact of the degree of stenosis on the number of MES and on embolus positivity was also evaluated using a Kruskal-Wallis one way ANOVA. For these analyses, the 12 patients with ICA occlusion were excluded. Significance was assumed at a value of $p<0.05$.

\section{Results}

The distribution of degrees of stenosis and presence of symptoms is given in the figure.

Thirty plaques were uniformly echolucent, 34 plaques were predominantly echolucent, 37 plaques were predominantly echogenic, and four were uniformly echogenic. In 46 patients a cross flow via the anterior communicating artery was present. Contralateral carotid artery high grade stenosis was present in nine patients, 12 patients had a contralateral ICA occlusion. No intracranial ICA or MCA stenoses were detected. Out of the 40 patients with either oral or intravenous anticoagulation, all but two had a stenosis $\geqslant 0 \%$.

The total number of MES was 212, ranging from $0-38$ in the individual patients. Twenty one out of 64 symptomatic patients (33\%) and four out of 41 asymptomatic patients (10\%) showed MES. Symptomatic patients were more likely to have emboli $(\mathrm{p}=0.007)$ than asymptomatic patients. The number of MES was higher in the symptomatic patients (mean 3.1 (SD 7.7)) compared with the asymptomatic patients (mean 0.3 (SD 1.0); $\mathrm{p}=0.006$ ). These values refer to the whole groups of patients, not only those embolus positive.

Positivity for MES and the number of MES increased with increasing degree of stenosis; there were no MES in patients with $\leqslant 80 \%$ stenosis ( $\mathrm{p}=0.015$ and $\mathrm{p}=0.019$, respectively).

There was a decline of MES positivity and of the number of MES with the time after the 
Studies on microembolus detection in carotid artery stenosis

\begin{tabular}{|c|c|c|c|c|c|c|}
\hline Authors & Year & $\begin{array}{l}\text { Degree of } \\
\text { stenosis (\%) }\end{array}$ & Asymptomatic & MES (\%) & Symptomatic & MES (\%) \\
\hline Siebler et $a l^{88}$ & 1994 & $\geqslant 70$ & 56 & 17 & 33 & 82 \\
\hline Georgiadis et $a l^{29}$ & 1994 & $>70$ & Not specified & Not specified & 13 & 92 \\
\hline Ries et al ${ }^{30}$ & 1995 & $\geqslant 60$ & 18 & 0 & 36 & 19 \\
\hline van Zuilen et al ${ }^{19}$ & 1995 & $\geqslant 70$ & & & 36 & 56 \\
\hline Valton et $a l^{14}$ & 1995 & Not specified & 28 & 14 & 20 & 20 \\
\hline Eicke et $a l^{31}$ & 1995 & $\geqslant 75$ & 9 & 0 & 13 & 38 \\
\hline Markus et $a l^{17}$ & 1995 & $\geqslant 30$ & 28 & 4 & 38 & 34 \\
\hline \multirow[t]{3}{*}{ Present study } & 1999 & $20-100$ & 41 & 10 & 64 & 33 \\
\hline & & $70-99$ & 29 & 14 & 41 & 41 \\
\hline & & 100 & 2 & 0 & 10 & 40 \\
\hline
\end{tabular}

ischaemic event; only one patient showed MES more than 80 days after the index event.

Echogenicity of the lesion did not affect either the number or presence of MES ( $p=0.56$ and $\mathrm{p}=0.49$, respectively). Eight patients out of 30 with plaques uniformly echolucent had MES (mean number of MES for the whole group of 30 patients 0.97 (SD 2.82), 10 patients out of 34 with plaques predominantly echolucent showed MES (mean number of MES 1.91 (SD 4.11)), seven out of 37 patients with predominantly echogenic plaques showed MES (mean number of MES 3.19 (SD 9.21)), and none of the four patients with uniformly echogenic plaques.

Out of the 25 patients with MES, four had possibly competing embolic sources. Two of these patients were in atrial fibrillation and two patients had a cross flow via the anterior communicating artery with a high grade carotid artery on the contralateral side.

\section{Discussion}

The degree of stenosis and the presence of ocular or cerebral ischaemic events were associated with a higher number and with a higher presence of MES in the present study. Therefore, our study suggests that the presence and number of clinically silent circulating MES may be surrogate parameters for macroembolic (stroke) risk. Valton et al described a nonsignificant trend towards a higher number of MES with increasing degree of stenosis. ${ }^{14}$ Babikian et al described a higher prevalence of MES in patients with a stenosis of more than $50 \%$ compared with patients with $\leqslant 50 \%$ stenosis. ${ }^{15}$ The table gives a summary of the studies performed so far in this field.

Our finding that echogenicity of the plaque is not related to an increased number of MES reflects the debated role of echolucency as a risk factor for stroke. ${ }^{1-616}$

The decline of the MES number with time has also been reported by other authors. ${ }^{17-19}$ The low frequency of MES after about 80 days may indicate the time needed for plaque healing after disruption. ${ }^{20-22}$

The presence of MES in a few asymptomatic patients suggests that clinically silent circulating microemboli may give additional information on the impending embolic potential of carotid artery stenoses not covered by the patient's history. At present, there is still insufficient evidence to operate on clinically asymptomatic stenoses only relying on the presence of MES. So far no large study has unequivocally shown MES to be a risk factor for stroke, as the number of patients is high and the observation time needed is long. ${ }^{23}$ Our study indirectly further supports this possible relation of microembolic events and macroembolic risk. We showed an increase in microembolic risk with increasing degree of stenosis and presence of symptoms, a relation which is well known for macroembolic risk from clinical endarterectomy trials. ${ }^{24-27}$

1 Bock RW, Lusby RJ. Carotid plaque morphology and interpretation of the echolucent lesion. In: Labs $\mathrm{KH}$, et al,
eds. Diagnostic vascular ultrasound. London: Edward Arnold, 1992:225-36.

2 el Barghouty N, Nicolaides A, Bahal V, et al. The identification of the high risk carotid plaque. Eur $\mathcal{F}$ Vasc Endovasc Surg 1996;11:470-8.

3 European Carotid Plaque Study Group. Carotid artery plaque composition: relationship to clinical presentation and ultrasound B-mode imaging. Eur $\mathcal{f}$ Vasc Endovasc Surg 1995;10:23-30.

4 Golledge J, Cuming R, Ellis M, et al. Carotid plaque characteristics and presenting symptoms. Br f Surg 1997;84: 1697-701.

5 Kardoulas DG, Katsamouris AN, Gallis PT, et al. Ultrasonographic and histologic characteristics of symptom-free and symptomatic carotid plaque. Cardiovasc Surg 1996;4:580-90.

6 Steffen CM, Gray Weale AC, Byrne KE, et al. Carotid artery atheroma: ultrasound appearance in symptomatic and asymptomatic vessels. Aust NZ F Surg 1989;59:529-34.

7 Spencer MP, Thomas GI, Nicholls SC, et al. Detection of middle cerebral artery emboli during carotid endarterectomy using transcranial Doppler ultrasonography. Stroke 1990;21:415-23.

8 Spencer MP. Detection of cerebral arterial emboli. In: Newell DW, et al, Transcranial Doppler. New York: Raven, 1992: 216-30

9 Droste DW, Dittrich R, Hermes S, et al. Four-gated transcranial Doppler ultrasound in the detection of circulating microemboli. Eur f Ultrasound 1999;(in press).

10 de Bray JM, Baud JM, Dauzat M. Consensus concerning the morphology and the risk of carotid plaques. Cerebrovasc Dis 1997;7:289-96.

11 de Bray JM, Glatt B. Quantification of atheromatous stenosis in the extracranial internal carotid artery. Cerebrovasc Dis 1995;5:414-26.

12 Görtler M, Widder B, Schuetz U. Quantifying medium- and high-grade carotid artery stenosis by ultrasound. Fournal d'Echographie et de Médecine Ultrasonore 1996;17:235-9.

13 Carpenter JP, Lexa FJ, Davis JT. Determination of duplex Doppler ultrasound criteria appropriate to the North American symptomatic carotid endarterectomy trial. Stroke
1996;27:695-9.

Valton L, Larrue V, Arrue P, et al. Asymptomatic cerebral embolic signals in patients with carotid stenosis. Correla-
tion with appearance of plaque ulceration on angiography. tion with appearance of
Stroke $1995 ; 26: 813-15$.

15 Babikian VL, Hyde C, Pochay V, et al. Clinical correlates of high-intensity transient signals detected on transcranial Doppler sonography in patients with cerebrovascular Doppler sonography in patie

16 Langsfield M, Grey-Weale AC, Lusby RJ. The role of plaque morphology and diameter reduction in the development of new symptoms in asymptomatic carotid arteries. F Vasc Surg 1989;9:548-57.

17 Markus HS, Thomson ND, Brown MM. Asymptomatic cerebral embolic signals in symptomatic and asymptomatic carotid artery disease. Brain 1995;118:1005-11.

18 Siebler M, Sitzer M, Rose G, et al. Silent cerebral embolism caused by neurologically symptomatic high-grade carotid stenosis. Brain 1993;116:1005-15.

19 van Zuilen EV, Moll FL, Vermeulen FE, et al. Detection of cerebral microemboli by means of transcranial Doppler monitoring before and after carotid endarterectomy. Stroke 1995;26:210-13.

20 Bornstein NM, Norris JW. The unstable carotid plaque. Stroke 1989;20:1104-6.

21 Fisher M, Blumenfeld AM, Smith TW. The importance of plaque disruption and hemorrhage. Arch Neurol 1987;44: $1086-9$

22 Sillesen $\mathrm{H}$, Nielsen $\mathrm{T}$. Clinical significance of intraplaque hemorrhage in carotid artery disease. F Neuroimaging 1998; 8:15-19.

23 Siebler M, Sitzer M, Rose G, et al. Cerebral microembolism and the risk of ischemia in asymptomatic high-grade internal carotid artery stenosis. Stroke 1995;26:2184-6.

24 North American Symptomatic Carotid Endarterectomy Trial Collaborators. Beneficial effect of carotid endarterectomy in symptomatic patients with high grade carotid stenosis. N Engl f Med 1991;325:445-53.

25 Executive Committee for the asymptomatic carotid atherosclerosis study. Endarterectomy for asymptomatic carotid artery stenosis. $\mathscr{F A M A}$ 1995;273:1421-8.

26 Barnett HJM. An update on NASCET and ECST. In: Branchereau A, et al. New trends and developments in carotid Branchereau A, et al. New trends and developments in carotid
artery disease. Armonk NY: Futura, 1998:107-16.

27 Farrell B, Fraser A, Sandercock P, et al. Randomised trial of endarterectomy for recently symptomatic carotid stenosis: final results of the MRC European carotid surgery trial (ECST). Lancet 1998;351:1379-87. 
28 Siebler M, Kleinschmidt A, Sitzer M, et al. Cerebral microembolism in symptomatic and asymptomatic high-grade

, Gross

29 Georgiadis D, Grosset DG, Quin RO, et al. Detection of intracranial emboli in patients with carotid disease. Eur $\mathcal{F}$ Vasc Surg 1994;8:309-14.
30 Ries F, Tiemann K, Pohl C, et al. High-resolution emboli detection and differentiation by characteristic postembolic
spectral patterns. Stroke 1998;29:668-72.

31 Eicke BM, von Lorentz J, Paulus W. Embolus detection in different degrees of carotid disease. Neurol Res 1995; 17:11-4.

\section{HISTORICAL NOTE}

The fissure of Rolando

The gyri of the brain are thought to have been first named "coils" by Praxagoras of Cos c 300 BC and by Erasistratus c260 BC. Vesalius amplified the description. ${ }^{1}$

Thomas Willis believed that: "movement is initiated in the cerebrum ...."."

Vicq d'Azyr described the convolutions in 1786 noting the differences in morphology in different animals. Magendie similarly had noticed that: "The number, the volume, the disposition of the circumconvolutions are variable ...."3

The attachment of eponyms to cerebral fissures started with the fissure of Sylvius in 1663 , though it had been clearly noted by Casper Bartholin (1585-1629), an anatomist in Denmark. The second structure to be so dignified was the fissure of Rolando in 1839

Rolando observed the precentral and postcentral gyri on either side of the great central fissure. He referred to the convolutions as enteroid processes, a term not very different from that of Erasistratus who compared them to the coils of the intestines. Rolando experimented on pigs, guinea pigs, goats, and sheep. He trepanned the cranium and applied an electric current through a wire placed in different parts of the brain. He observed violent contractions, then stupor; but because of the crude local damage that he knew he had caused, he was reluctant to draw firm conclusions about the relation of function to the anatomical areas examined.

"I rarely obtained a constant effect since it is extremely difficult to recognize what bundles of fibres have been lacerated . . . it is not possible to determine the consequences clearly and distinctly ..."

Indeed many of his experiments were directed not at the cortex but at the cerebellum, striatum, thalamus, and quadrigeminal bodies. Rolando found the largest motor responses by stimulation of the cerebellum and stressed the importance of this structure in determining movement.

Pierre Flourens (1794-1867) in refuting much of Rolando's work ${ }^{4}$ was accused of plagiarism; he therefore gave an annotated French translation to disprove these charges. Flourens experimented mainly on birds. $\mathrm{He}$ believed that the cerebrum was not divided functionally, but that intelligence and special senses were diffusely distributed throughout the brain: an early landmark of the holistic hypotheses of brain function.

It fell to Francois Leuret to give credit to Rolando: "Between these two convolutions exists a furrow that separates them ...; it is as constant as the Sylvian fissure. I have called this furrow the fissure of Rolando, because it was this anatomist who first described it in man, in whom it is still more developed than in the monkey."

Vicq d'Azyr had illustrated the central sulcus in 1786, but attached no special importance to it, so Rolando deserves the credit for its demonstration, despite the experimental difficulties he honestly recounted.

Luigi Rolando was born in Turin. After completing his medical studies he soon concentrated on experimental anatomy. $\mathrm{He}$ studied in Florence and became physician to the King of Savoy in Turin. The invasion of Italy by Napoleon drove him into exile in Sardinia for 12 years, where he became Professor of "Theoricopractical" Medicine at Sassari. $\mathrm{He}$ returned to Turin as Professor of Anatomy. ${ }^{6}$
Rolando worked extensively on the cerebellum. He observed minor damage to cause staggering, but complete destruction caused total locomotor paralysis in his animals. $\mathrm{He}$ therefore regarded the cerebellum as of major importance in determining not only the regulation but also the strength of movement. Although an inaccurate view, this was a great step forwards from the prevailing notions of the cerebellum as controller of intellect, sensation, or vital forces.

He was also responsible for demonstrating in unstained tissue, "a particular grey matter in the posterior third of the posterior horns", which he noticed was, "more gelatinous ... of a different colour ..."

The substantia gelatinosa was subsequently named after this remarkable anatomist. An original engraving of Luigi Rolando is found in the Biblioteque de l'Academie Nationale de Medecine, Paris

J M S PEARCE

304 Beverley Road, Anlaby,

Hull HU10 7BG, UK

1 Vesalius A. De humani corporis fabrica. Basle: 1543:130. Translated in: Clarke E, O'Malley 1543:130. Translated in: Clarke E, O'Malley
CD. The human brain and spinal cord. 2nd ed. San Francisco: Norman, 1996:386.

2 Willis T. 1661-2. Cited by: Dewhurst K. Willis's Oxford lectures. Oxford: Sandford, 1980

3 Magendie FJ. An elementary compendium of physiology; for the use of students. Translated by: E Milligan. Philadelphia: James Webster, 1824: 104

4 Rolando L. Daggio sopra la vera struttura del cervello dell'uomo e degl'animali e sopra le funzioni del sistem nervoso. Sassari: Stampa Privileg, 1809. Cited in: Webb Haymaker, Francis Schiller, eds. The founders of neurology, 2nd ed. Springfield: Charles C Thomas, 1970: 73-6.

5 Leuret F. Translated by: MV Anker. Anatomie comparée du système nerveux. Paris: Baillière, 1839;1:397-8.

6 Fadiga, In: Belloni, ed. Essays on the history of Italian neurology. Milan: Ist di Storia della Medicine, 1963:204. 